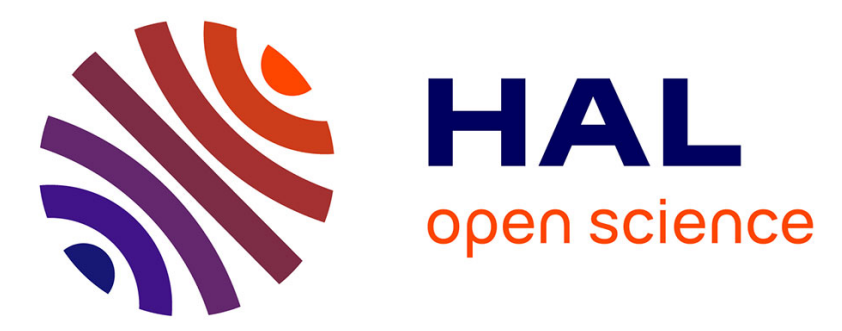

\title{
SCATTERING BY COMPLEX POTENTIALS WITHOUT COMPLEX TRAJECTORIES
}

\author{
F. Guillod, P. Huguenin
}

\section{To cite this version:}

F. Guillod, P. Huguenin. SCATTERING BY COMPLEX POTENTIALS WITHOUT COMPLEX TRAJECTORIES. Journal de Physique Colloques, 1984, 45 (C6), pp.C6-27-C6-33. 10.1051/jphyscol:1984604 . jpa-00224205

\section{HAL Id: jpa-00224205 https://hal.science/jpa-00224205}

Submitted on 1 Jan 1984

HAL is a multi-disciplinary open access archive for the deposit and dissemination of scientific research documents, whether they are published or not. The documents may come from teaching and research institutions in France or abroad, or from public or private research centers.
L'archive ouverte pluridisciplinaire HAL, est destinée au dépôt et à la diffusion de documents scientifiques de niveau recherche, publiés ou non, émanant des établissements d'enseignement et de recherche français ou étrangers, des laboratoires publics ou privés. 


\section{SCATTERING BY COMPLEX POTENTIALS WITHOUT COMPLEX TRAJECTORIES}

F. Guillod and P. Huguenin

Institut de Physique de L'Université, me Breguet 1, CH-2000 Neuchâtel, Switzerland

Résume - Nous rappelons rapidement le concept d'absorption en mécanique classique et nous montrons que l'analogue quantique est un potentiel imaginaire proportionnel à $\hbar$. Les déphasages quantiques correspondant à ce potentiel sont calculés asymptotiquement à l'ordre $\hbar^{2}$.

Abstract - We briefly recall the concept of absorption in classical mechanics and we show that the quantum analogue is an imaginary potential proportional to $\hbar$. The quantum phase shifts corresponding to this potential are calculated asymptotically to order $\hbar^{2}$.

\section{ABSORPTION IN CLASSICAL DYNAMICS}

The concept of absorption is not contained in classical system dynamics. In this case, the state of the system is described by a point $x$ in the appropriate phase space E. No probability concept allows to speak about any kind of decay law of the system itself.

The appropriate framework is classical statistical mechanics. The state is a probability density $W t$ in the phase space. Without absorption and for Hamiltonian dynamics we have the Liouville (sometimes called vlasov) equation

$$
\frac{\partial}{\partial t} W_{t}=\left\{h, W_{t}\right\}
$$

where $h$ is the Hamiltonian and $\{$,$\} the Poisson bracket. This reversible equation$ insures conservation of the total probability. If $\phi_{t}$ is the Hamiltonian flow [1] associated to $h$, the solutions of eq. (1) may be written as

$$
W_{t}(x)=W_{0}\left(\phi_{-t}(x)\right) \text {. }
$$

The current $J_{t}$ is related to $\phi_{t}$ in such a way that the current flow lines coincide with the orbits of $\phi_{t}$ :

$$
J_{t}(x)=\dot{x} W_{t}(x)=\dot{\phi}_{t} \circ \phi_{-t}(x) W_{t}(x) .
$$

In this scheme the knowledge of $J_{t}$ is sufficient to predict the change of the state. 
The velocity field

$$
\dot{x}(x)=\{x, h\}=\dot{\phi}_{t} \circ \phi_{-t}(x)
$$

is independent of $t$ if $h$ is. We have

$$
\nabla \cdot J_{\ell}=\left\{W_{\ell}, h\right\}
$$

and eq. (1) is a continuity equation.

In order to describe absorption we have to modify the evolution equation (1). We put

$$
\frac{\partial}{\partial t} W_{t}(x)+\nabla \cdot J_{t}(x)=-a(x) W_{t}(x) \quad, a(x) \geqslant 0 .
$$

$a(x)$ is the absorption rate. It has to be positive if the system disappears and does not spontaneously create itself! The current $J_{t}$ is given by (3). Its evolution depends not only on $J_{t}$ itself but also on $a(x)$.

Defining the total derivative, we obtain $[2,3]$

$$
\frac{d}{d t} W_{t}(x)=\frac{\partial}{\partial t} W_{t}(x)+\dot{x} \nabla W_{t}(x)=\frac{\partial}{\partial t} W_{t}(x)+\nabla \cdot J_{t}(x)=-a(x) W_{t}(x) .
$$

For an "observer" dragged along the Hamiltonian flow lines, the time derivative of the density $W_{t}$ is given by $-a(x) W_{t}(x)$. We can give a closed expression for this evolution :

$$
W_{t}\left(\phi_{t}(y)\right)=W_{0}(y) \exp \left(-\int_{0}^{t} d t^{\prime} \alpha\left(\phi_{t} \cdot(y)\right)\right) .
$$

Or in a form similar to (2)

$$
W_{t}(x)=W_{0}\left(\phi_{-t}(x)\right) \exp \left(-\int_{0}^{t} d t^{\prime} a\left(\phi_{t^{\prime}} \circ \phi_{-t}(x)\right)\right) .
$$

This is the expected result : absorption does not change the orbits in phase space. simply, the probability is decreased by an exponential factor which we intexpret as shadow. We have neither reflexion nor diffraction effects which are related to the wave nature of the dynamics.

We underline the classical picture without complex trajectories. We simply add a probability notion to the unchanged orbits. The absorption is described by $a(x)$, a function with a dimension inverse of time.

\section{QUANTIZATION}

We want a quantum analogue to eq. (5). Clearly, at time $t$, the state of the Gibbs ensemble is described by a state operator $W_{t}$, the Hamilton's function for the reversible part of the evolution is replaced (by traditional recipe) by the Hamiltonian $\mathrm{H}$ and the Poisson bracket by the commutator. In this way, Liouville equation is transcribed into a von Neumann's form. With the same recipes, we built a symmetrical operator corresponding to a :

$$
a \longmapsto A=A^{+}
$$


Because $W_{t}$ is hermitian, we have to take the anticommutator of $A$ and $W_{t}$. Finally,

$$
\frac{\partial}{\partial t} W_{t}=\frac{1}{i \hbar}\left[H, W_{t}\right]-\frac{1}{2}\left[A, W_{t}\right]_{t} \quad, A \geqslant 0 .
$$

positivity of a is replaced by positivity of the operator $A$. Taking the trace :

$$
\frac{\partial}{\partial t} \operatorname{Tr} W_{t}=-\operatorname{Tr}\left(A W_{t}\right) \leqslant 0 \text {. }
$$

Like a, A has dimension inverse of time. Starting with a normalized pure state, eq. (8) gives new unnormalized pure states. The equation separates nicely. If we put

$$
W_{t}=\left|\Psi_{t}\right\rangle\left\langle\psi_{t}\right|
$$

we see that if

$$
i \hbar \frac{\partial}{\partial t}\left|\psi_{t}\right\rangle=H\left|\psi_{t}\right\rangle-\frac{i \hbar}{2} A\left|\psi_{t}\right\rangle,
$$

than (10) fulfils (8).

For a single particle in a potential $V(\vec{q})$ and an absorption independent of momentum, we can introduce a complex potential

$$
V(\vec{q})=V(\vec{q})-\frac{i \hbar}{2} a(\vec{q}) \text {. }
$$

The important point is that the imaginary part of the potential is proportional to $\hbar$, i.e. it vanishes in the classical limit.

Putting

$$
\psi_{l}(\vec{q})=\left\langle\vec{q} \mid \psi_{t}\right\rangle=\sqrt{\rho} \exp \left(\frac{i}{\hbar} s\right)
$$

we obtain the Schroedinger equation

$$
\text { ih } \frac{\partial}{\partial t} \Psi_{t}(\vec{q})=\left[-\frac{\hbar^{2}}{2 m} \Delta+V(\vec{q})-\frac{i \hbar}{2} \alpha(\vec{q})\right] \psi_{t}(\vec{q})
$$

Following Madelung we obtain for $\rho$ and $S$ the coupled equations

$$
\begin{aligned}
& \vec{\nabla}\left(\frac{1}{m} \rho \vec{\nabla} s\right)+\frac{\partial}{\partial t} \rho=-\alpha \rho \\
& \frac{1}{2 m}(\vec{\nabla} s)^{2}+V-\frac{\hbar^{2}}{2 m} \frac{1}{\sqrt{\rho}} \Delta \sqrt{\rho}+\frac{\partial}{\partial t} S=0 .
\end{aligned}
$$

Corrections to the classical behaviour are contained in the so-called quantum potential of Madelung [4]

$$
V_{\text {OUnM Tum }}(\vec{q})=-\frac{\hbar^{2}}{2 m} \frac{1}{\sqrt{\rho(\vec{q})}} \Delta \sqrt{\rho(\vec{q})} \text {. }
$$

This term is physically important in regions where the probability density changes markedly on distances of the order of the de Broglie's wave length. In particular, caustics (turning points) deserve special considerations. 


\section{QUALITATIVE DISCUSSION}

Equations (15) closely correspond to the classical equation (5) of solution (7). Simply, we stay now in the configuration space

$$
\rho_{t}(\vec{q})=\int d^{3} p W_{t}(\vec{q}, \vec{p})
$$

and the Hamiltonian flow $\phi_{t}$ is described by the generating function $S_{t}(\vec{q})$ [5]. Assuming we neglect the quantum potential, the effect of $\alpha(\vec{q})$ is simply a darkening of some parts of the bundle of classical trajectories forming the wave $\psi_{t}$. It appears borders between light and shadow.

Now, such borders are quantum mechnically unstable. In order to see this, we calculate a simple model. Assume a profile (one dimensional)

$$
\begin{aligned}
& \rho(q)=\frac{1}{4}\left(1-t h \frac{q}{q_{0}}\right)^{2} \rho_{0} \\
& V_{\text {ounawiun }}(q)=-\frac{h^{2}}{m q_{0}} \cdot \frac{t h \frac{q}{q_{0}}}{\operatorname{ch} \frac{q}{q_{0}}} \cdot \exp \left(\frac{q}{q_{0}}\right)
\end{aligned}
$$

This potential acts like a knife which repels the trajectories from the border. The trajectories bundle is qualitatively modified. Crossing of trajectories gives rise to interference patterns.

This is exactly what happens in diffraction. The two light spots interpretation of Wallace [6] for diffraction scattering supports the same view. The use of eq. (15) may provide an evaluation for the centroids of the spots. This has not been done yet.

\section{THE SCATTERING PROBLEM}

In the specific case of scattering by a spherical symmetric potential, the physical information is contained in the phase-shift. Its real part is a generating function for time-delay and scattering angle. The imaginary part describes attenuation. our goal is the exact calculation of the phase-shifts to the order $\hbar^{2}$ [7]. At fixst sight, it may appear hopeless. Alxeady without potential the centrifugal barrier generates caustics where quantum effects are important. For this reason, we use a comparison method where the fxee case is calculated exactly. We extend the Rosen and yennie method $[8]$ to a complex potential. In this case the radial part of eq. (14) reads

$$
E u=-\frac{h^{2}}{2 m} u^{\prime \prime}+\left[V(r)-i W(r)+\frac{h^{2}(l(+1)}{2 m r^{2}}\right] u
$$

where $u$ is related to $\psi_{t}$ by

$$
\psi_{t}(\dot{q})=Y_{e m}(\theta, \varphi) \frac{u(r)}{r} \exp \left(-\frac{i}{t} E t\right)
$$


and $W=2 \hbar a$ where a does not depend on $\hbar$. In this way we get a finite absorption even in the classical limit.

In all asymptotic calculations we would like variables whose range extends from $-\infty$ to $+\infty$. To this end we perform the Langer [9] transformation

$$
r=\frac{\hbar}{\sqrt{2 m \varepsilon}} \exp \left(\frac{\sqrt{2 m \varepsilon}}{\hbar} \rho\right), u(r)=w(\rho) \exp \left(\frac{\sqrt{2 m \varepsilon}}{2 \hbar} \rho\right)
$$

The new variable $\rho$ runs from $-\infty$ to $+\infty$. For $\rho=0$ we are at a distance $\lambda$ from the scattering center. We obtain from eq. (18) :

$$
\hbar^{2} \omega^{\prime \prime}+\exp \left(\frac{2 \sqrt{2 m E}}{\hbar} \varphi\right)\left[p^{2}(\rho)+i \hbar m a(\rho)\right] \omega=0
$$

with

$$
p^{2}(r)=2 m\left(\varepsilon-\frac{\lambda^{2}}{2 m r^{2}}-V(r)\right) \quad, \lambda=\hbar\left(\rho+\frac{1}{2}\right) .
$$

We shall obtain an approximate solution of eq. (21) by using the comparison method of Miller and Good [10]. We put

$$
\omega(\rho)=\alpha(\rho) \omega_{0}(\sigma(\rho) / \hbar)+\beta(\rho) \omega_{0}^{\prime}(\sigma(\rho) / \hbar)
$$

where $\omega_{0}$ is a solution of the free particle equation

$$
\omega_{0}^{4}(\sigma / \hbar)+\exp \left(\frac{2 \sqrt{2 m E}}{\hbar} \sigma\right) \cdot p_{0}^{2}(\sigma) \cdot \omega_{0}(\sigma / \hbar)=0
$$

where $p_{0}$ is the local radial momentum in the free case $V=0 . \omega_{0}$ is a RiccatiBessel function of argument $\exp \left(\frac{\sqrt{2 m E}}{\hbar} \sigma\right)$.

our problem is now to calculate $\alpha, \beta$ and $\sigma$ in some appropriate way. substituting (23) in (21) we obtain a linear combination between $\omega_{0}$ and $\omega_{0}^{\prime}$. Equating the coefficients independently to zero we have :

$$
\begin{aligned}
& \hbar^{2} \alpha^{\prime \prime}+i \hbar m a \exp \left(\frac{2 \sqrt{2 m \varepsilon}}{\hbar} \rho\right) \alpha+\left[\exp \left(\frac{2 \sqrt{2 m E}}{\hbar} \rho\right) p^{2}-\exp \left(\frac{2 \sqrt{2 m E}}{\hbar} \sigma\right) p_{0}^{2}\left(\sigma^{\prime}\right)^{2}\right] \alpha=2 \hbar \exp \left(\frac{\sqrt{2 m E}}{\hbar} \sigma\right) p \sqrt{\sigma^{\prime}}\left[\exp \left(\frac{\sqrt{2 m E}}{\hbar} \sigma\right) p \cdot \sqrt{\sigma^{\prime}} \beta\right]^{\prime} \\
& \hbar^{2} \beta^{\prime \prime}+i \hbar m \alpha \exp \left(\frac{2 \sqrt{2 m E}}{\hbar} \rho\right) \beta+\left[\exp \left(\frac{2 \sqrt{2 m E}}{\hbar} \rho\right) p^{2}-\exp \left(\frac{2 \sqrt{2 m E}}{\hbar} \sigma\right) p_{0}^{2}\left(\sigma^{\prime}\right)^{3}\right] \beta=-2 \hbar \sqrt{\sigma^{\prime}}\left[\sqrt{\sigma^{\prime}} \alpha\right]^{\prime} .
\end{aligned}
$$

We simplify the problem by annulating the square bracket :

$$
\exp \left(\frac{\sqrt{2 m E}}{\hbar} \rho\right) p(\rho)=\exp \left(\frac{\sqrt{2 m E}}{\hbar} \sigma\right) p_{0}(\sigma) \sigma^{\prime} \text {. }
$$

This is a differential equation for $\sigma(\rho)$. We choose the integration constant in such a way that $\sigma$ represents a coordinate, i.e. $\sigma$ has to be real : the first zeros (from outside) of $p^{2}(\beta)$ and $p_{0}^{2}(\sigma)$ have to coincide. At this turning point

$$
\sigma_{t}\left(\rho_{t}\right)=\frac{\hbar}{\sqrt{2 m \varepsilon}} \log \left(\frac{\lambda}{\hbar}\right) \text {. }
$$


Now the simplified system (25) allows an expansion in powers of $h$ :

$$
\begin{aligned}
& \alpha(\rho)=\alpha_{0}+\alpha_{1} \hbar+\alpha_{2} \hbar^{2}+\cdots \\
& \beta(\rho)=\beta_{0}+\beta_{1} \hbar+\beta_{2} \hbar^{2}+\cdots .
\end{aligned}
$$

For example, in first order

$$
\begin{aligned}
& i m \alpha \exp \left(\frac{2 \sqrt{2 m E}}{\hbar} \rho\right) \alpha_{0}=2 \exp \left(\frac{\sqrt{m E}}{\hbar} \sigma\right) p_{0} \sqrt{\sigma^{\prime}}\left[\exp \left(\frac{\sqrt{2 m E}}{\hbar} \sigma / p_{0} \sqrt{\sigma^{\prime}} \beta_{0}\right]^{\prime}\right. \\
& i m \alpha \exp \left(\frac{2 \sqrt{2 m E}}{\hbar} \rho\right)\left(\beta_{0}=-2 \sqrt{\sigma^{\prime}}\left[\sqrt{\sigma^{\prime}} \alpha_{0}\right]^{\prime} .\right.
\end{aligned}
$$

We verify that for arbitrary $G_{0}=$ cst:

$$
\begin{aligned}
& \alpha_{0}=\frac{G_{0}}{\sqrt{\sigma^{\prime}}} \cos \left[i \int_{\rho_{t}}^{\rho} d x \frac{m \alpha}{2 p} \exp \left(\frac{\sqrt{2 m E}}{\hbar} x\right)\right] \\
& \beta_{0}=\frac{G_{0}}{p \sqrt{\sigma^{\prime}}} \exp \left(-\frac{\sqrt{2 m E}}{\hbar} \sigma\right) \sin \left[i \int_{\rho_{t}}^{\rho} d x \frac{m \alpha}{2 p} \exp \left(\frac{\sqrt{2 m E}}{\hbar} x\right)\right]
\end{aligned}
$$

is a solution which goes to $\alpha_{0}=c s t$ and $\beta_{0}=0$ for $a=0$. The next approximation gives

$$
\begin{aligned}
& \alpha_{1}=\frac{1}{2}\left(\alpha_{0} I_{1}-\exp \left(\frac{\sqrt{2 m E}}{\hbar} \sigma\right) p_{0} \beta_{0} I_{2}\right) \\
& \beta_{1}=\frac{1}{2}\left(\beta_{0} I_{1}+\exp \left(-\frac{\sqrt{2 m E}}{\hbar} \sigma\right) p_{0}^{-1} \alpha_{0} I_{2}\right)
\end{aligned}
$$

with

$$
I_{1}=\frac{1}{G_{0}^{2}} \int_{\rho_{t}}^{\rho} d x\left[\alpha_{0}^{\prime \prime} \beta_{0}-\beta_{0}^{\prime \prime} \alpha_{0}\right] \quad, I_{2}=\frac{1}{\sigma_{0}^{2}} \int_{\rho_{t}}^{\rho} d x\left[\alpha_{0}^{\prime \prime} \alpha_{0} \exp \left(-\frac{\sqrt{2 m \varepsilon}}{h} \sigma\right) \bar{p}_{0}^{-1}+\beta_{0}^{\prime \prime} \beta_{0} \exp \left(\frac{\sqrt{2 m \varepsilon}}{h} \sigma\right) p_{0}\right] .
$$

Along these lines we are able to extract the phase shift from the behaviour of (23) at infinity. The result is

$$
\begin{aligned}
\delta_{l}=\delta_{l}(W K B) & +i \hbar \int_{r_{t}}^{\infty} d r \frac{m a}{2 p}+\hbar^{2}\left\{-\frac{1}{24} \int_{r_{t}}^{\infty} d r \frac{1}{r p}\left[\frac{4(E-V)-5 r V^{\prime}-r^{2} V^{\prime \prime}}{2(E-V)-r V^{\prime}}\right]^{\prime}+\right. \\
& \left.+\frac{m^{2}}{8} \int_{r_{t}}^{\infty} d r\left[\frac{a^{2}}{p^{3}}-\frac{a_{z}^{2}}{\left[\left(r-r_{t}\right)\left(p_{l}^{2}\right)^{)^{3}}\right]^{3 / 2}}\right]\right\}+O\left(\hbar^{3}\right)
\end{aligned}
$$

with

$$
a_{t}=a\left(r_{t}\right), \quad\left(p_{t}^{2}\right)^{\prime}=\left(p^{2}\right)^{\prime}\left(r_{t}\right)
$$

and

$$
\delta_{e}(W K B)=\int_{r_{i}}^{\infty} d x\left[\sqrt{2 m(E-V)-\frac{\lambda^{2}}{x^{2}}}-\sqrt{2 m E}\right]-\sqrt{2 m E} r_{t}+\frac{\pi}{2} \lambda
$$


This result is compatible with the qualitative considerations. In first order, absorption gives rise to an imaginary contribution to $\delta_{\ell}, i \cdot e$ attonuation. In second order we obtain a contribution to the scattering angle.

The discussion of the result is obscured by the fact that the customary form of the scattering amplitude as a sum over integral values of $l$ is a quantum mechanical expression which depends also on $\hbar$.

In ref. [7] we discuss specific examples.

\section{REEERENCES}

[1] ARNOLD V., Méthodes mathênatiques de la mécanique classique (Editions de Moscou 1974).

(2) AMTET J.-P., HUGUENIN P., Mécaniques classique et quantique dans l'espace de phase (Université de Neuchâtel 1981).

[3] HUGUenin P., Dissipation et physique quantique dans "La pensée physique contemporaine", Ed. A. Fresnel (1982) 223.

[4] Madelung E., Zeit. für Physik 40 (1926) 322.

[5] AMIET J.-P., hUGUENIN P., Helv. Phys. Acta 55 (1982) 278.

[6] WALLACE S.J., Dynamics of hadron nucleus interactions in Proceedings of the Conference on High Energy Physics and Nuclear Structure, versailles (1981) 203 c. HugueniN P., Phys. Lett. 7OA (1979) 372.

[7] GUILLOD F., HUGUENIN P., Phys. Lett. A, in press.

[8] ROSEN M., YENNIE D.R., J. Math. Phys. 5 (1964) 1505.

[9] LANGER R.E., Phys, Rev. 51 (1937) 669.

[10] MILLER S.C., GOOD R.H., Phys. Rev. 91 (1953) 174. 